\title{
A Modified Lunar Reconnaissance Orbiter (LRO) High Gain Antenna (HGA) Controller Based on Flight Performance
}

\author{
Neerav Shah \\ Attitude Control System Engineering Branch \\ NASA Goddard Space Flight Center \\ Greenbelt, MD
}

\begin{abstract}
The National Aeronautics and Space Administration's (NASA) Lunar Reconnaissance Orbiter (LRO) was launched on June 18, 2009 and is currently in a $50 \mathrm{~km}$ mean altitude polar orbit around the Moon. LRO was designed and built by the NASA Goddard Space Flight Center in Greenbelt, MD. The spacecraft is three-axis stabilized via the attitude control system (ACS), which is composed of various control modes using different sets of sensors and actuators. In addition to pointing the spacecraft, the ACS is responsible for pointing LRO's two appendages, the Solar Array (SA) and the High Gain Antenna (HGA). This study reviews LRO's HGA control system. Starting with an overview of the HGA system, the paper delves into the single input single output (SISO) linear analysis followed by the controller design. Based on flight results, an alternate control scheme is devised to address inherent features in the flight control system. The modified control scheme couples the HGA loop with the spacecraft pointing control loop, and through analysis is shown to be stable and improve transient performance. Although proposed, the LRO project decided against implementing this modification.
\end{abstract}

\section{INTRODUCTION}

Lunar Reconnaissance Orbiter (LRO), tasked with mapping the lunar surface, was launched on June 18, 2009 aboard an Atlas V launch vehicle from Cape Canaveral, FL [1]. LRO was designed and built by the NASA Goddard Space Flight Center in Greenbelt, MD. The spacecraft is three-axis stabilized via the attitude control system (ACS), which is composed of various control modes using different sets of sensors and actuators. The ACS is responsible for maintaining attitude in all phases of the mission, starting with launch vehicle separation through cruise, orbit and mission insertion, commissioning, and nominal mission. LRO is currently in its $50 \mathrm{~km}$ mean altitude polar orbit around the Moon and has been collecting great science with its suite of seven instruments. In addition to pointing the spacecraft properly, the ACS team is responsible for pointing LRO's two appendages, the Solar Array (SA) and the High Gain Antenna (HGA).

\section{HIGH GAIN ANTENNA SYSTEM}

The HGA is a double-gimbaled device extended on a deployable arm, and has to point at a specified Earth ground station to within 1.5 degrees. Figure 1 is a mechanical drawing of LRO with the Spacecraft and HGA coordinate systems defined [2]. As can be seen, the HGA coordinate system is defined with both gimbals at their home positions (i.e., at 0 angle). The double arrow-heads indicate the positive direction of rotation for the gimbals. Each of the two HGA gimbals has a 180 degree range of motion, from -90 degrees to 90 degrees. 


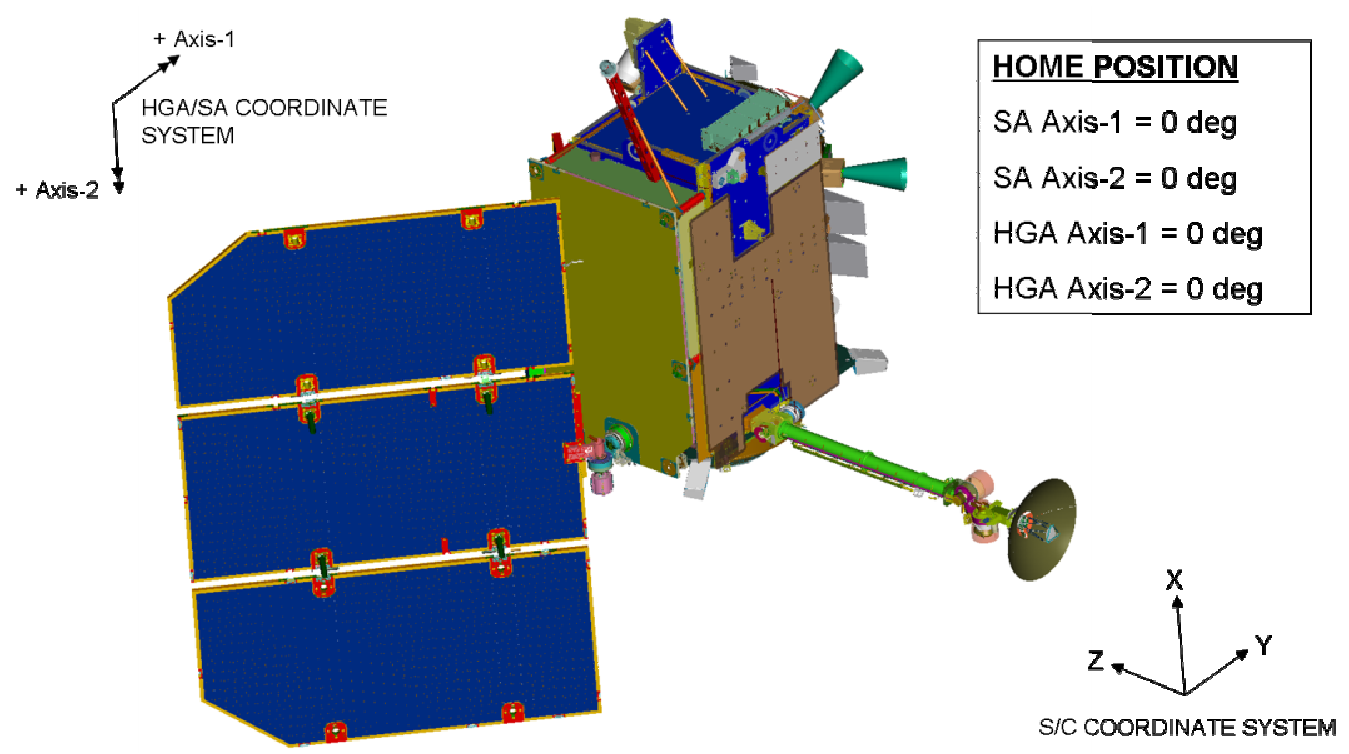

Figure 1. LRO Mechanical Drawing with SC and HGA/SA Axes

\subsection{REQUIREMENTS}

The 1.5 degree pointing requirement must be satisfied in the presence of mechanical errors in the structure, gimbals, calibration errors, and algorithm accuracy. Algorithm accuracy is the portion of the error budget that has the largest impact on the HGA control design, and is allocated $42 \operatorname{arcsec}$ (asec) or 1 motor step per axis root sum squared (RSS) in steady-state. The primary level 4 (subsystem-level) requirement on the HGA control system is: the HGA controller shall not adversely affect the SC pointing controller. In addition to these requirements, all control systems shall have a $6 \mathrm{~dB}$ gain margin and 30 degrees phase margin, a NASA Goddard "Gold Rule" [3].

\subsection{HARDWARE}

LRO's High Gain Antenna (HGA) system is composed of a main dish, an outer motor, an inner motor extended on a deployable arm, and a gimbal control electronics (GCE) box.

\subsubsection{STEPPER MOTOR}

Starsys developed the stepper motors for LRO. Each of the gimbals is a permanent magnet 3-phase harmonic drive stepper motor. Each motor cardinal step is equivalent to 0.0075 degrees on the output through a 1:200 gear reduction ratio. One of the key features of this motor is that it has micro-step capability. Micro-stepping enables the gimbal electronics to electronically control the motor during a step, thereby reducing the jitter caused by the motor moving a cardinal step [4].

Figure 2 shows the rotation convention for the gimbals, which define the double arrows and coordinate system in Figure 1. 


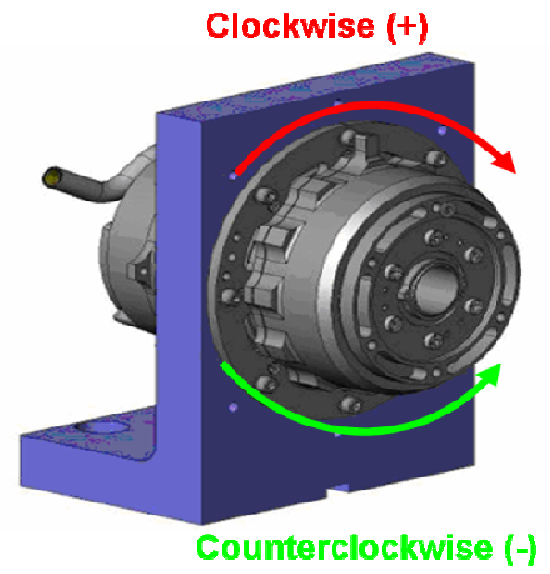

Figure 2. HGA Gimbal with rotation convention

\subsubsection{GIMBAL CONTROL ELECTRONICS (GCE)}

Broadreach Engineering developed the gimbal control electronics (GCE) for LRO. The stepper motors are mechanical devices, so there needs to be control electronics to interface with the flight software. The GCE provides that interface. LRO's GCE is considered "smart," since it offers different methods of controlling the motors [5].

The GCE offered the following different commands:

- Move: Angle command

- Track: Rate and direction command

- Go: Rate command with pre-defined location

- Stop: Stop all motion

- Initialize: Find home and reset encoder

Interfacing through this GCE using different commands provides for different control strategies.

\section{FLIGHT HIGH GAIN ANTENNA CONTROL SYSTEM}

Flight operations and interactions between the HGA and the spacecraft drive the HGA control design. During nominal operation, the spacecraft is maintaining a lunar nadir attitude in "Observing mode," the primary sciencecollecting attitude control mode. To provide communication, the HGA must maintain a near-constant Earthtracking rate in both gimbals. Due to this constant rate tracking, the GCE "Track" command is used in a feedback control scheme. The Track command shapes the physics of the problem - with current gimbal angle as the measurement and desired gimbal rate as the command; defining the plant as a single-integrator.

A functional control diagram is shown in Figure 3. The motor angle is fed through negative feedback to determine a gimbal angle error. A desired, or target gimbal angle is computed through an inverse kinematics algorithm based on: spacecraft ephemeris, Earth ephemeris, and spacecraft attitude [6]. A target attitude is used instead of the onboard estimated attitude to decouple the HGA and spacecraft control loops. This is done to avoid any possible coupling issues as well as to simplify the control design to be a single-input single-output During nominal operations, the spacecraft is orbiting at a nominal velocity about the moon (orbit rate). Therefore, this known rate is fed forward through the HGA control, allowing the controller to adjust the gimbal rate about the nominal orbit rate. Since the steady-state pointing requirement is tight, a PI controller is implemented for each of the two HGA axes. 


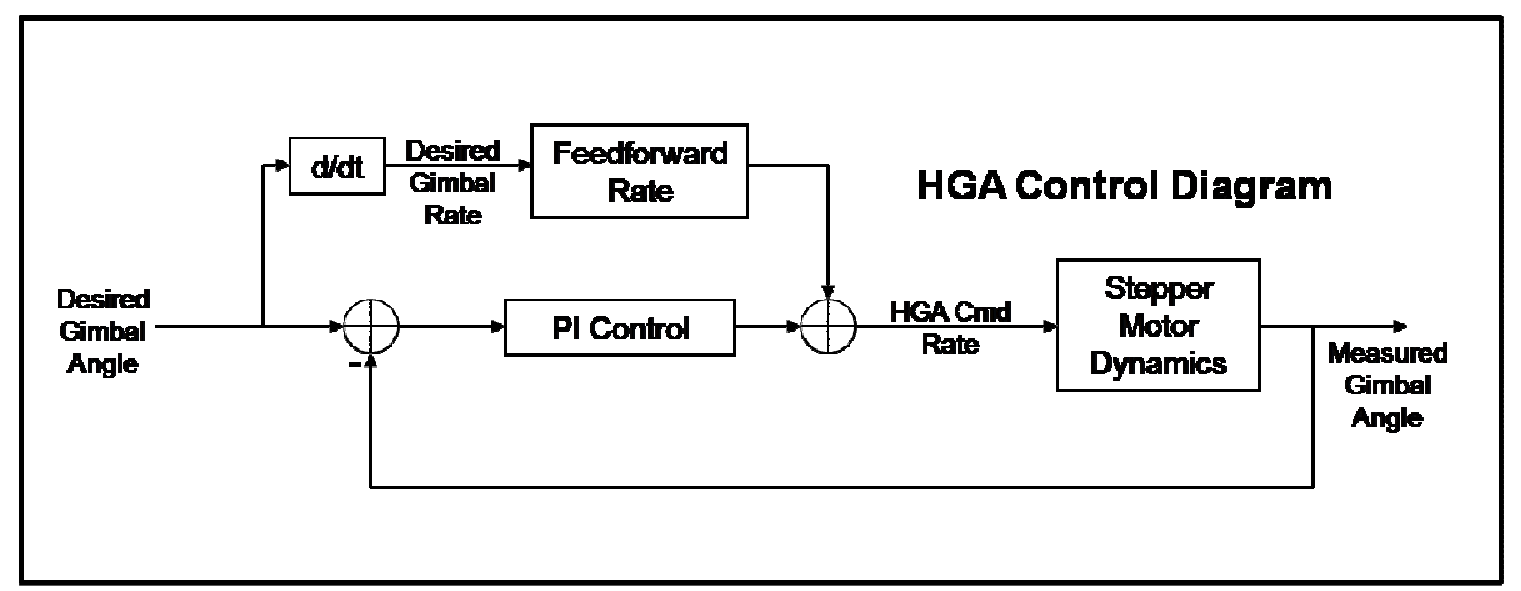

Figure 3. HGA Functional Control Diagram

\subsection{ANALYSIS}

Each gimbal motor is treated as a separate single input single output (SISO) system, so a classical design technique is used to meet the gain and phase margin requirements [7][8].

The plant transfer function (stepper motor) is given by:

$$
G_{p}=\frac{1}{s}
$$

The PI controller transfer function is given by:

$$
G_{P I}=K_{P}+\frac{K_{I}}{s}
$$

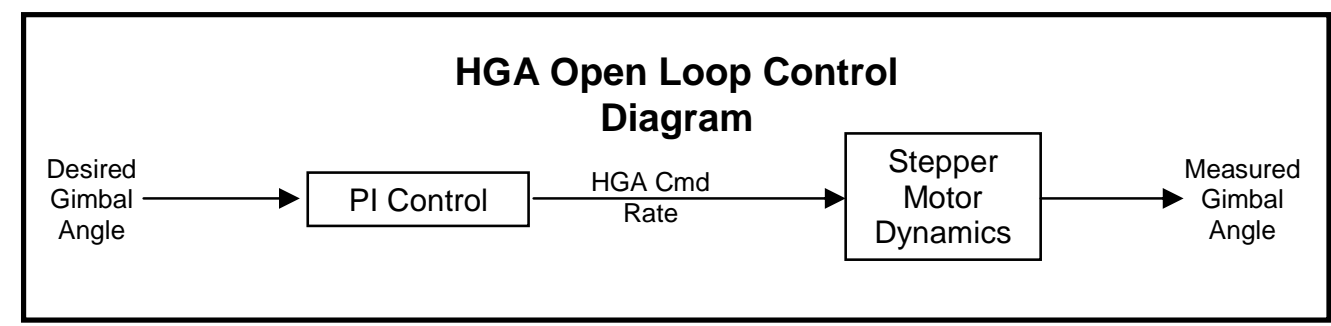

Figure 4. HGA Open Loop Control Diagram

Based on these two transfer functions and inspection of Figure 4, the open loop transfer function is given by:

$$
G_{O L}=G_{P I} \cdot G_{P}=\frac{K_{P} s+K_{I}}{s^{2}}
$$

The rate feed forward term of Figure 3, is given by:

$$
G_{F F}=K_{F F} s
$$

Equations (1) through (4) along with Figure 3 give the closed loop transfer function: 


$$
G_{C L}=\frac{\theta_{m}}{\theta_{d}}=\frac{K_{F F} s^{2}+K_{P} s+K_{I}}{s^{2}+K_{P} s+K_{I}}
$$

From Equation (5), the closed loop poles are computed as:

$$
s=-\frac{K_{P}}{2} \pm \frac{1}{2} \sqrt{K_{P}{ }^{2}-4 \cdot K_{I}}
$$

And the closed loop zeros are computed as:

$$
z=-\frac{1}{2} \frac{K_{P}}{K_{F F}} \pm \frac{1}{2 \cdot K_{F F}} \sqrt{K_{P}{ }^{2}-4 \cdot K_{F F} \cdot K_{I}}
$$

For closed loop stability, we need to ensure all poles have negative real parts [7][8].

\title{
3.2. CONTROLLER DESIGN
}

The HGA controller gains $\mathrm{K}_{\mathrm{P}}, \mathrm{K}_{\mathrm{FF}}$, and $\mathrm{K}_{\mathrm{I}}$, as discussed in Equations (1) through (7) are selected to meet performance and stability requirements. The system is modeled in Matlab/Simulink using discrete versions of Equations (1) through (5). A time delay of one ACS control cycle is added to the Simulink model to represent processor lag. An iterative design approach (loop-shaping) is taken utilizing the Bode plot, Nichols charts, and Step response as benchmarks of performance, to determine the gains.

The flight HGA controller has the following characteristics (refer to Figure 5 and Figure 6 for the Bode plot, Nichols chart and Step response, respectively of the HGA Control System):

\author{
Bandwidth: $0.023 \mathrm{~Hz}$ \\ Gain Margin: $32.4 \mathrm{~dB}$ \\ Phase Margin: $78.5 \mathrm{deg}$ \\ Settling Time: $115 \mathrm{sec}$
}

Gain Margin and Phase Margin requirements are clearly met. The low bandwidth $(0.023 \mathrm{~Hz})$ ensures that this controller does not interfere with the spacecraft controller, which has a bandwidth of $0.1 \mathrm{~Hz}$. Since there is a feed forward rate term, the PI controller does not have to have a high bandwidth because it will only be correcting small perturbations about the desired rate.
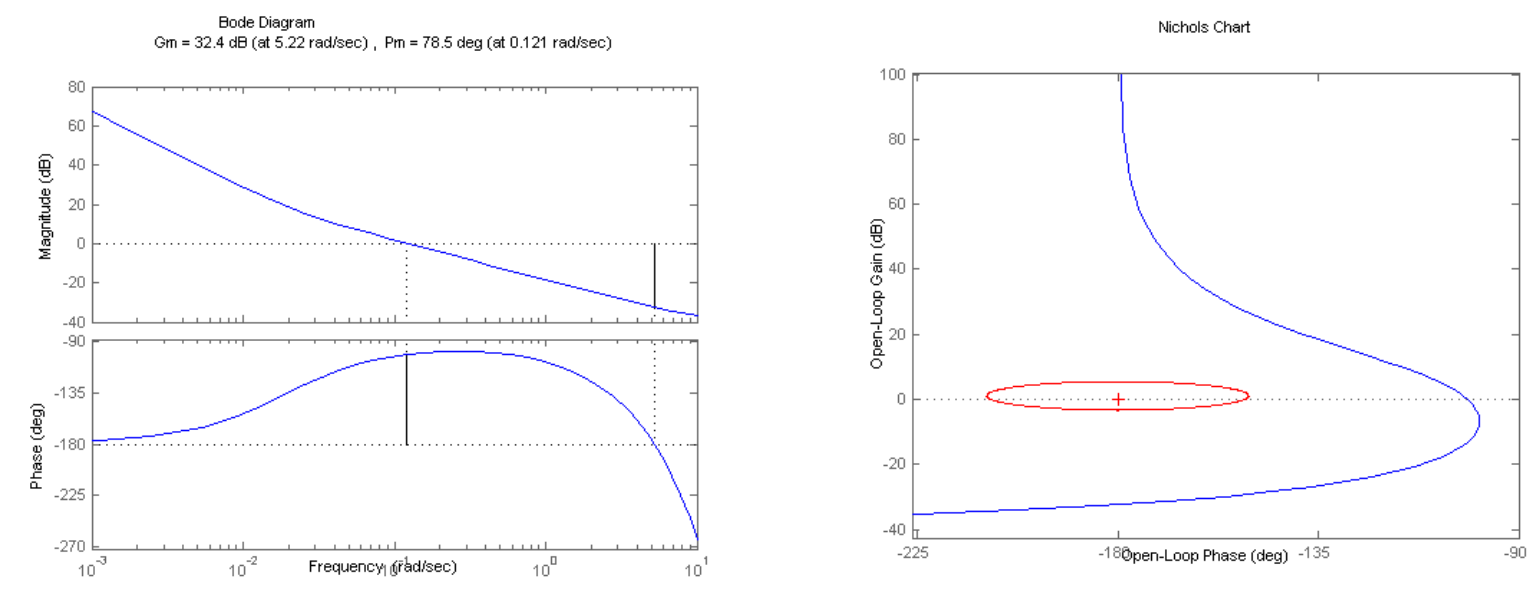

Figure 5. Bode Plot and Nichols Plot of HGA Controller - Red circle indicates GM and PM requirement 


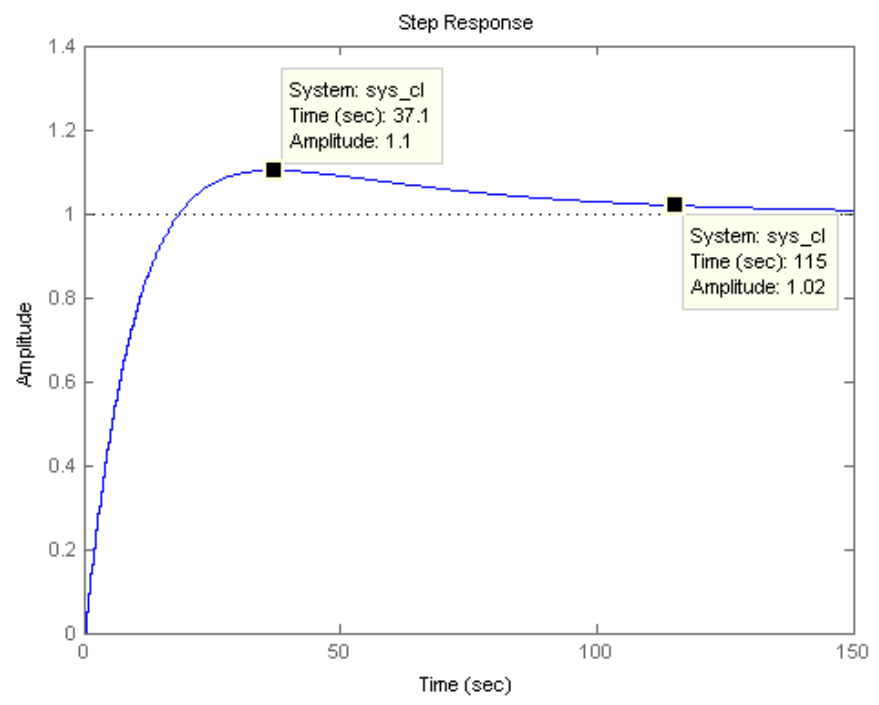

Figure 6. Closed-Loop Step Response of HGA Control System

\section{FLIGHT PERFORMANCE}

Flight results of LRO's HGA controller show acceptable performance - i.e., communication is maintained with sufficient link margin. However, during large spacecraft attitude maneuvers (slew), communication and thus telemetry are lost. This is a result of the HGA moving to its target faster than the spacecraft (spacecraft max slew rate in Observing mode is $0.1 \mathrm{deg} / \mathrm{sec}$ and HGA max rate is $0.5 \mathrm{deg} / \mathrm{sec}$ ). An attitude maneuver is executed via command changes to an offset attitude, which changes the target attitude instantaneously, making the spacecraft slew. Instantaneously changing the target attitude changes the HGA target angles instantaneously as well. Since the spacecraft and HGA do not have the same maneuver rate, communication is lost. This loss of communication during a large attitude maneuver is a known feature of the control design and is due to the HGA target computation based on a target attitude, rather than the actual estimated attitude.

Although acceptable, the loss of communication during a slew, and thus a loss of spacecraft telemetry, is undesirable and therefore needs to be addressed. The remainder of the paper details an alternate control scheme that seeks to resolve the inherent design feature of the flight HGA controller.

\section{MODIFIED HIGH GAIN ANTENNA CONTROL SYSTEM}

An alternate control scheme that does not require a flight software patch is desirable. Therefore, the only design tools available are changes to flight parameters that are loaded to the spacecraft via tables. These tables include parameters such as: HGA controller gains, spacecraft controller gains, and spacecraft structural filter coefficients. There is also a parameter that allows the HGA controller to select between using a target spacecraft attitude or an estimated spacecraft attitude as a source to the inverse kinematics algorithm. By selecting the estimated spacecraft attitude to compute the desired HGA angles, the loss of communication during large attitude maneuvers is averted. The reason is because the HGA target is computed based on the actual attitude of the spacecraft instead of a target spacecraft attitude. An undesirable effect is that the HGA and spacecraft control loops become coupled.

The following analysis details the modified HGA control design using the estimated spacecraft attitude instead of the target spacecraft attitude. Since this couples the spacecraft controller and the HGA controller, there may be some spacecraft stability issues. If coupling the two control loops causes issues, then the HGA controller, the spacecraft Observing mode controller, the spacecraft structural filter, or a combination of all three, will have to be re-designed. 


\subsection{SPACECRAFT - HIGH GAIN ANTENNA COUPLED MODEL}

A SISO model is developed coupling the spacecraft and HGA control loops, refer to Figure 7 for a control diagram of the coupled control system. The spacecraft has three rotational degrees of freedom, and the HGA has two. If motion is considered in one axis of the spacecraft, then the HGA will move an equal distance in the opposite direction to maintain pointing. If the spacecraft rotates in two axes, then that rotation would be distributed between the two HGA degrees of freedom. Therefore, a worst case value for the "targeting" block that couples the spacecraft attitude to the HGA desired angle is -1 .

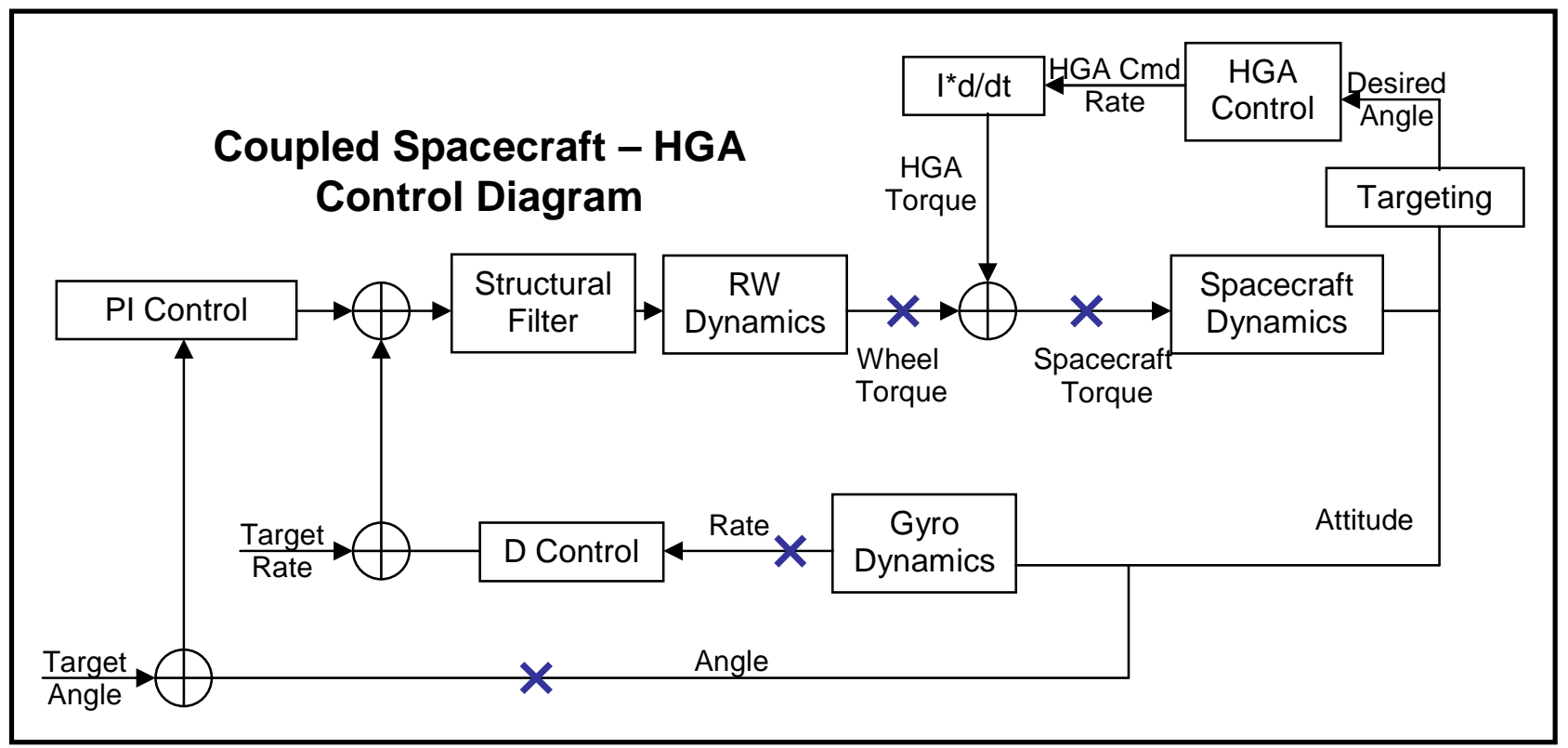

Figure 7. Coupled Spacecraft and High Gain Antenna Control Functional Diagram

As the HGA moves, it imparts a torque on the spacecraft. But, the HGA is an integrator plant with rate as an input and angle as an output. So, to determine the HGA torque, the commanded rate is differentiated and multiplied by the HGA inertia. In Section 3.1, the HGA control closed-loop transfer function is given from desired angle to measured angle. For the coupled model shown in Figure 7, the transfer function from desired angle to commanded rate is needed, refer to Figure 8.

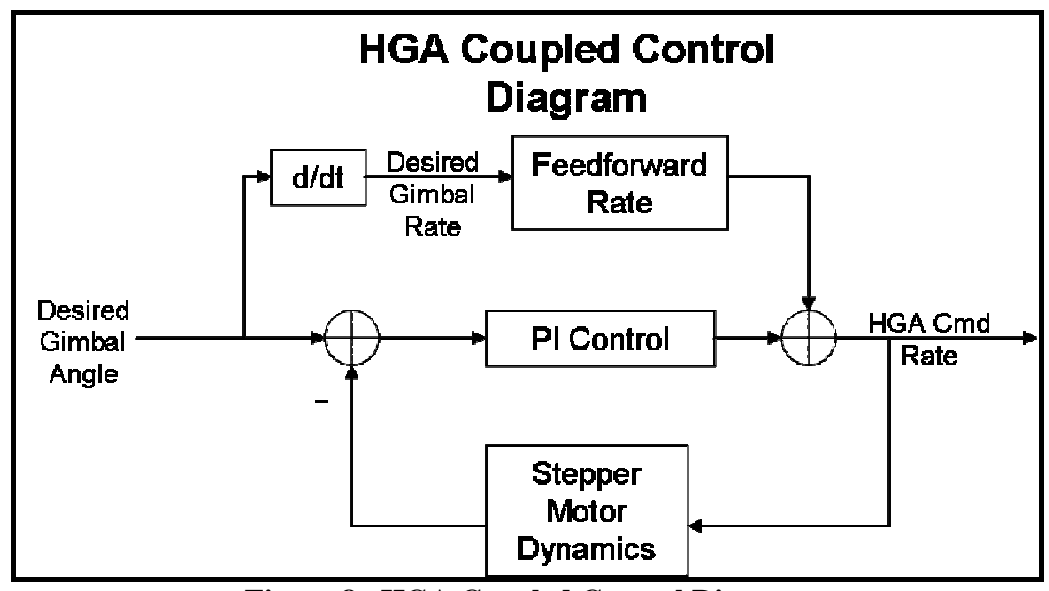

Figure 8. HGA Coupled Control Diagram 
The transfer function from desired angle to commanded rate is:

$$
\begin{gathered}
\left(1+\frac{K_{P}}{s}+\frac{K_{I}}{s^{2}}\right) \omega_{c m d}=\left(K_{F F} s+K_{P}+\frac{K_{I}}{s}\right) \theta_{d} \\
G_{H G A}=\frac{\omega_{c m d}}{\theta_{d}}=\frac{K_{F F} s^{3}+K_{P} s^{2}+K_{I} s}{s^{2}+K_{P} s+K_{I}}
\end{gathered}
$$

The HGA inertia can be found by using the parallel axis theorem [9]:

$$
I_{S C}^{H G A}=I_{H G A}^{H G A}+m^{H G A} \cdot\left(L_{S C}^{H G A}\right)^{2}
$$

where,

$I_{S C}^{H G A}$ : Inertia of the HGA system with respect to the Spacecraft reference frame

$I_{H G A}^{H G A}$ : Inertia of the HGA system with respect to the HGA reference frame

$m^{H G A}$ : Mass of HGA system lumped at HGA reference frame origin

$L_{S C}^{H G A}$ : Distance from Spacecraft reference frame origin to HGA reference frame origin

Substituting the values into Equation (8) gives:

$$
\begin{aligned}
& I_{S C}^{H G A}=0.5362 \mathrm{~kg} \cdot \mathrm{m}^{2}+8.77 \mathrm{~kg} \cdot(2.723 \mathrm{~m})^{2} \\
& I_{S C}^{H G A}=65.56 \mathrm{~kg} \cdot \mathrm{m}^{2}
\end{aligned}
$$

Coupling the HGA and spacecraft control loops results in differentiating the commanded HGA rate to obtain an applied torque. The HGA torque is applied to each of the three axes of the spacecraft, assuming a worst case interaction between the HGA and the spacecraft control loop.

\subsection{SPACECRAFT DYNAMICS MODEL}

In section 5.1, the coupling between the HGA and spacecraft is established. For stability analysis and design of the spacecraft controller, a 3-axis rigid body model with fuel slosh and the first 6 flexible body modes is used as the plant. K\&D Research's MultiBody Dynamics Analysis (MDA) Simulink Toolbox is used to design the system, and a state-space system is extracted through Matlab's 'dlinmod' feature.

In addition to the complex plant model, time delays, reaction wheel dynamics [10], gyro dynamics [11], and a structural filter are added to the control loop and modeled in Matlab/Simulink. The respective transfer functions are:

$$
\begin{gathered}
G_{r w}=\frac{\omega_{r w}}{s+\omega_{r w}} \\
G_{g y r o}=\frac{\omega_{g y r o}^{2}}{s^{2}+2 \cdot \zeta_{g y r o} \cdot \omega_{g y r o} \cdot s+\omega_{g y r o}^{2}} \\
G_{s f}=\frac{n_{0} s^{3}+n_{1} s^{2}+n_{2} s+n_{3}}{d_{0} s^{3}+d_{1} s^{2}+d_{2} s+d_{3}}
\end{gathered}
$$


where,

$\omega_{r w}$ is the reaction wheel bandwidth

$\omega_{\text {gyro }}$ is the rate gyro bandwidth

$\zeta_{\text {gyro }}$ is the rate gyro damping ratio

and the coefficients of the numerator and denominator for the structural filter are those of a $3^{\text {rd }}$ order elliptic filter.

\subsection{COUPLED SYSTEM LINEAR ANALYSIS}

By selecting the estimated spacecraft attitude to use in computing the HGA angles, the HGA and spacecraft control loops are coupled. To determine stability, of a complex SISO system with multiple loops, each loop is broken, the open loop transfer function is found, stability analysis is performed on the open loop, and then transient performance is determined from the closed loop system. For the spacecraft control (Observing mode) stability analysis (without HGA), the loop is broken at Angle, Rate and Wheel Torque. But, these breaks don't take into account the HGA minor loop. Therefore, an additional break is established at Spacecraft Torque (blue $\mathrm{x}$ marks a loop break in Figure 7). Bode and Nichols plot are created to determine stability merits and a step response is generated to determine closed loop performance, for each loop break and all three spacecraft axes.

Breaking the loop at angle, rate and wheel torque and performing stability analysis with and without the HGA couples resulted in marginal impact on phase margin, gain margin and modal suppression. When the loop is broken at the spacecraft torque, a larger impact is seen. Figure 9 shows a bode plot of the Observing mode control loop broken at the spacecraft torque for the x-axis with and without the HGA coupling. When the HGA is not coupled, the gain and phase margin are set at $0.4 \mathrm{rad} / \mathrm{sec}$ and $1 \mathrm{rad} / \mathrm{sec}$, respectively, and they meet the $6 \mathrm{~dB} / 30 \mathrm{deg}$ requirement. But, when the HGA is coupled, the gain and phase margin are now set at $9 \mathrm{rad} / \mathrm{sec}$ and $10 \mathrm{rad} / \mathrm{sec}$, respectively, and no longer meet the $6 \mathrm{~dB} / 30 \mathrm{deg}$ requirements. The resulting negative gain margin indicates instability. The structural filter ( $3^{\text {rd }}$ order elliptical), designed to depress the high frequency flexible modes, is negated when the HGA controller is coupled, indicating that the HGA control is interacting with the flexible modes of the spacecraft.

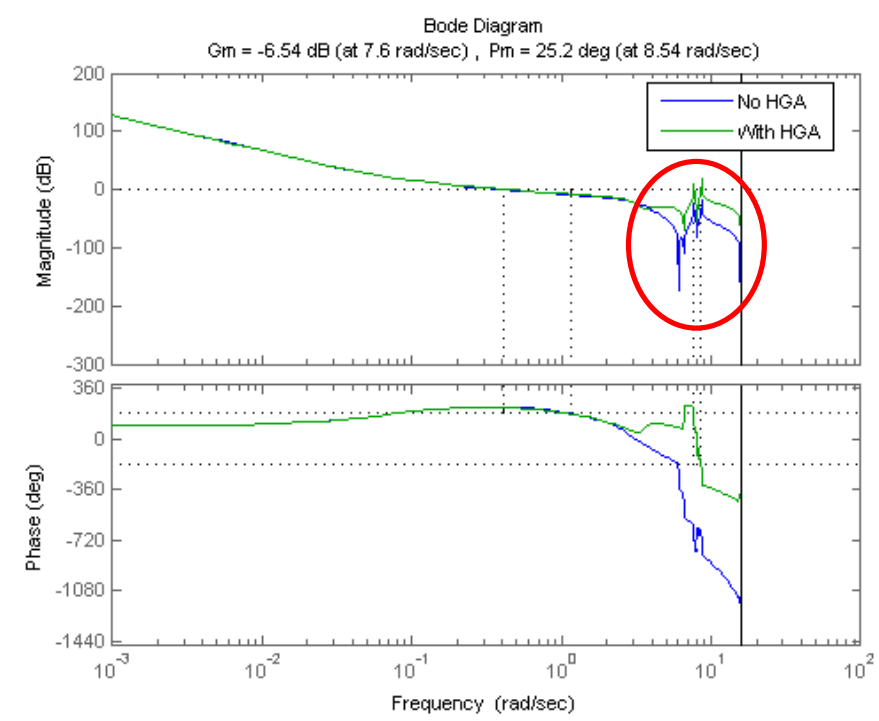

Figure 9. Bode plot of Observing mode controller broken at $x$-axis spacecraft torque; with and without HGA control loop

To gain additional insight, look at the Nichols plot for this case, Figure 10. The gain and phase margin is set by the $(180,0)$ point on the plot [7][8]. Looking at the green line (HGA coupled), it is indeed the high frequency modes that are setting the gain and phase margin, instead of the low frequency rigid body mode, as is desired. The high frequency modes cross the 0 -gain line, showing there is instability. 


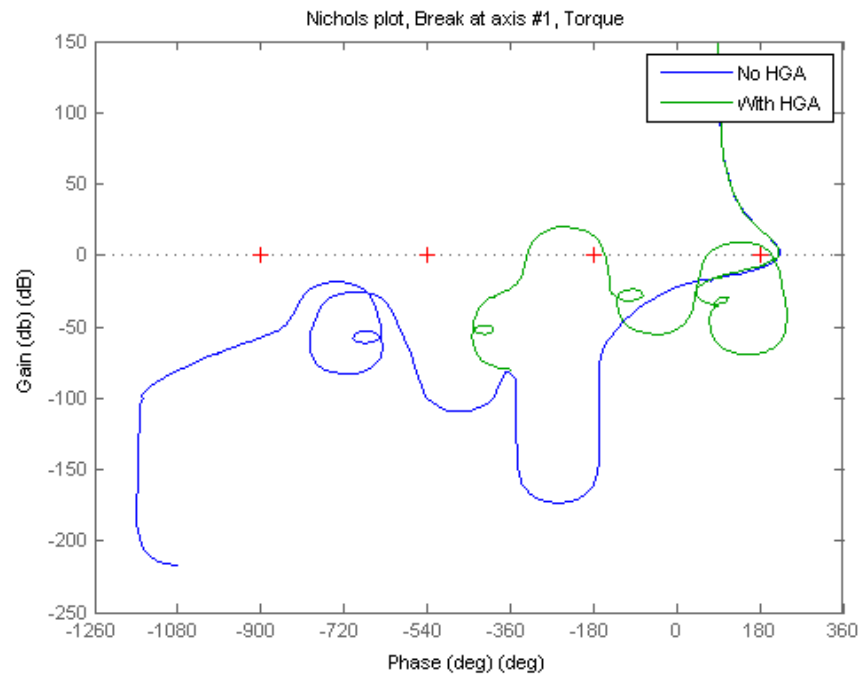

Figure 10. Nichols plot of Observing mode controller broken at $x$-axis spacecraft torque; with and without HGA control loop

From the Bode and Nichols plots, an unstable system is established. Looking at the step of the closed loop system will confirm this, refer to Figure 11. Without the HGA (blue line), the response settles, but with the HGA (green line), the response is oscillatory and unstable.

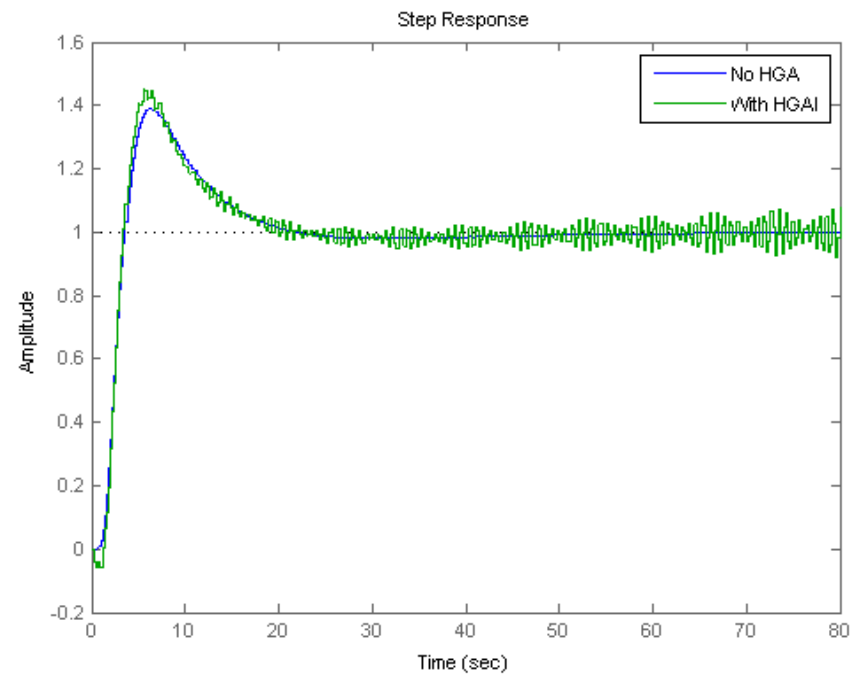

Figure 11. Step response of Observing mode controller broken at $x$-axis spacecraft torque; with and without HGA control loop

Selecting the estimated spacecraft attitude as the source of the HGA angles instead of the target spacecraft attitude, results in an unstable spacecraft control system. Redesigning the HGA controller, spacecraft Observing mode controller, structural filter, or a combination of the three is the next step.

\subsection{MODIFIED CONTROL FOR COUPLED SYSTEM}

Figure 9 through Figure 11 show that the HGA flight controller interacts with the flexible modes of the spacecraft. Since the spacecraft flexible modes cannot be changed, the HGA controller must be changed. 


\subsubsection{ANALYSIS}

To gain insight, examine the transfer functions of the coupled model. The simplified coupled system diagram, Figure 12, shows that the HGA control loop and spacecraft control loop can each be reduced to a single transfer function:

$$
\begin{gathered}
G_{H G A}=\frac{\tau_{H G A}}{\theta_{D}}=I_{S C}^{H G A} s \frac{K_{F F} s^{3}+K_{P} s^{2}+K_{I} s}{s^{2}+K_{P} s+K_{I}} \\
G_{S C c}=\frac{\tau_{w}}{\theta_{S C}}= \\
=\frac{\omega_{r w}\left[K_{p} s^{3}+\left(K_{d} \omega_{\text {gyro }}^{2}+2 K_{p} \zeta_{\text {gyro }} \omega_{\text {gyro }}+K_{i}\right) s^{2}+\left(K_{p} \omega_{\text {gyro }}^{2}+2 K_{i} \zeta_{\text {gyro }} \omega_{\text {gyro }}\right) s+K_{i} \omega_{\text {gyro }}^{2}\right]}{s^{4}+\left(2 \zeta_{\text {gyro }} \omega_{\text {gyro }}+\omega_{r w}\right) s^{3}+\left(\omega_{\text {gyro }}^{2}+2 \zeta_{\text {gyro }} \omega_{\text {gyro }} \omega_{r w}\right) s^{2}+\omega_{\text {gyro }}^{2} \omega_{r w} s}
\end{gathered}
$$

Combining the two transfer function to get one:

$$
G_{\text {Combined }}=G_{S C c}+G_{H G A}
$$

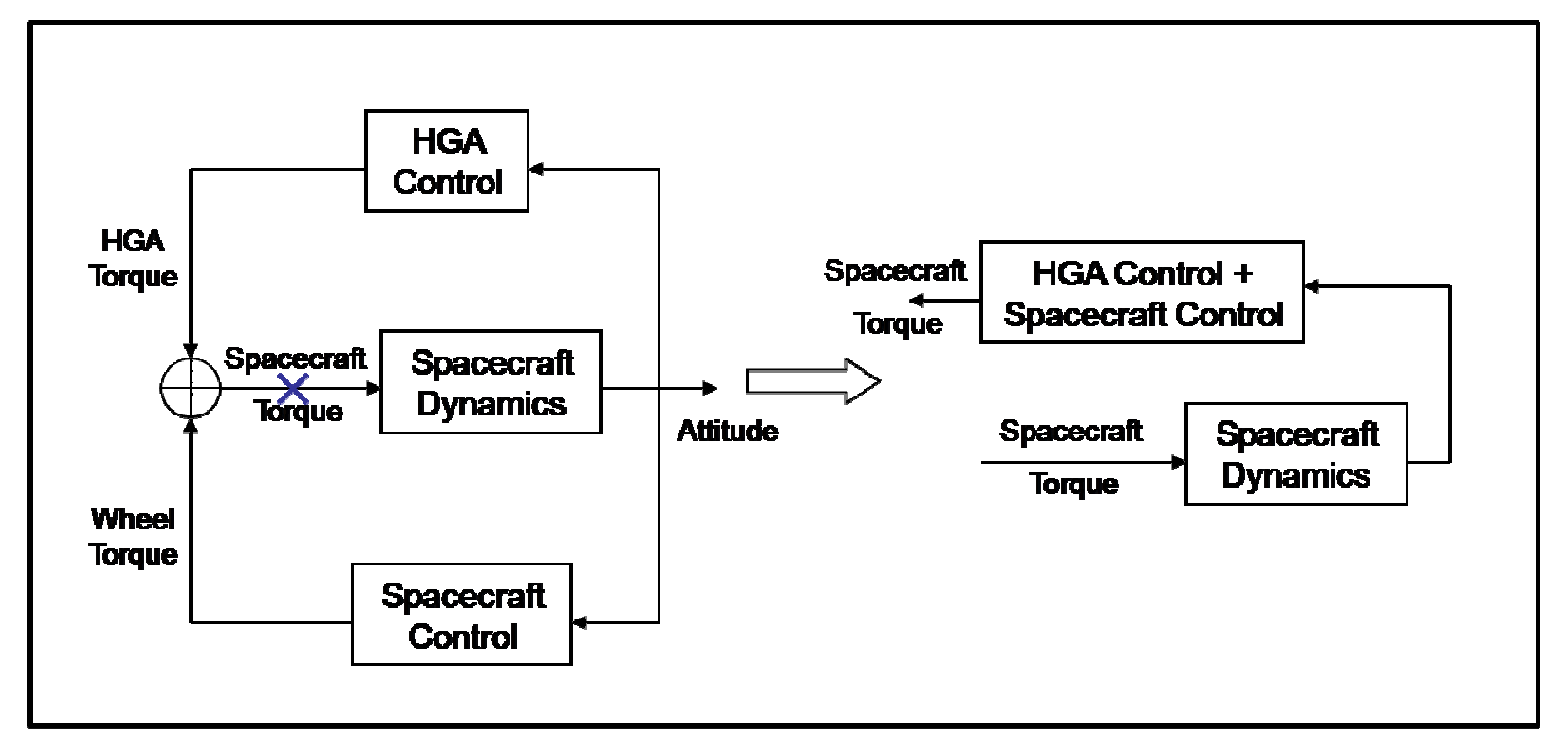

Figure 12. Simplified Spacecraft - HGA Control Diagram

The combined control transfer function is an 8th order polynomial in $s$ in the numerator and a 6th order polynomial in $s$ in the denominator (from inspection) - an improper transfer function.

The open loop transfer function is given by:

$$
\begin{aligned}
& G_{O L}=G_{\text {Combined }} \cdot G_{\text {spacecraft }} \cdot \\
& G_{O L}=f\left(\frac{I_{S C}^{H G A}}{I_{S C}^{S C}}\right)
\end{aligned}
$$

This yields an 8 th order polynomial in $\mathrm{s}$ in the numerator, but an $8^{\text {th }}$ order polynomial in $\mathrm{s}$ in the denominator $-\mathrm{a}$ proper transfer function. In addition, notice that this is a function of the inertia ratio of the HGA to the Spacecraft. Therefore, if the HGA inertia is small compared to the spacecraft, the impact of the HGA will be minimal. 
Although Equation (17) is a proper transfer function, Equation (16) and (14) are not. Proper fraction expansion can be used to make them proper, or they can be made proper by decreasing the order of the numerator.

\subsubsection{MODIFIED CONTROLLER DESIGN AND ANALYSIS}

To model the interaction between the HGA and the spacecraft, the commanded gimbal rate is differentiated and multiplied by inertia to get torque. This action is shown to result in an improper transfer function, Equation (14). From inspection of Equation (14), removing the feed forward rate from the HGA control loop, will make Equation (14) proper; i.e., set $\mathrm{K}_{\mathrm{FF}}=0$. Plotting the Bode plot, Nichols plot, and step response and comparing to the case where the HGA controller gains are not altered will help gauge whether this minor change will address the stability issues associated with coupling the HGA and spacecraft control loops.

Figure 13 through Figure 15 give the Bode plot, Nichols plot and closed loop step response of the nominal Observing mode controller (blue line), the Observing mode controller with HGA coupling (green line) and the Observing mode controller with modified HGA coupling, i.e., $\mathrm{K}_{\mathrm{FF}}=0$ (red line). Removing the feed forward rate from the HGA control loop has solved the stability issue associated with coupling the spacecraft and HGA controllers. But, the feed forward rate was used in conjunction with a 'low-effort' PI controller. Section 5.3 shows that the HGA flight controller has a very slow step response, which is acceptable because of the feed forward rate. But, without the feed forward rate, the PI controller is not sufficient. Therefore, the PI controller gains must be tuned to provide for increased performance.

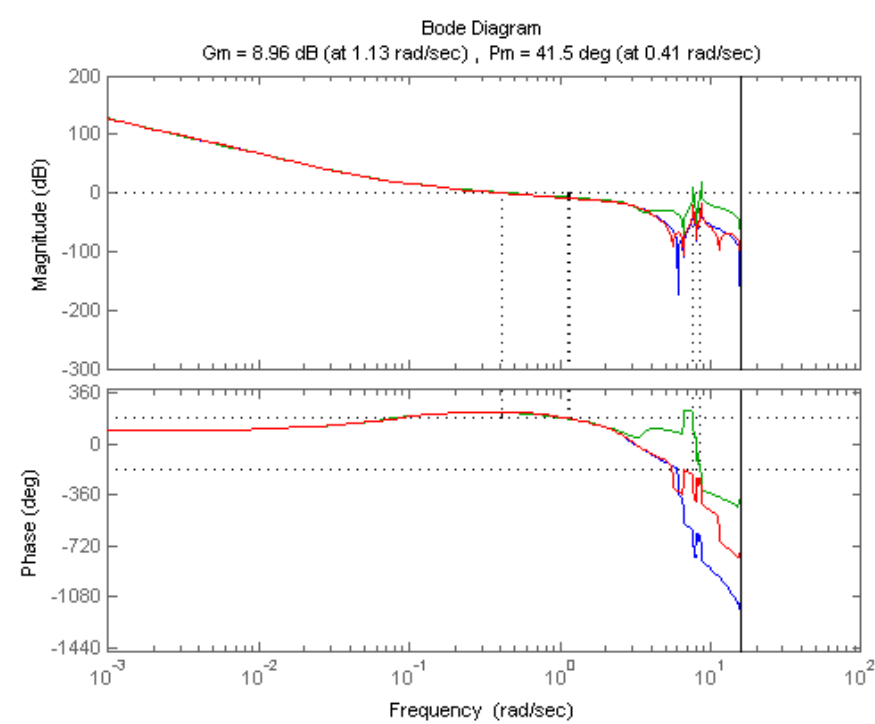

Figure 13. Bode plot of HGA Control with $\mathrm{K}_{\mathrm{FF}}=0$ 


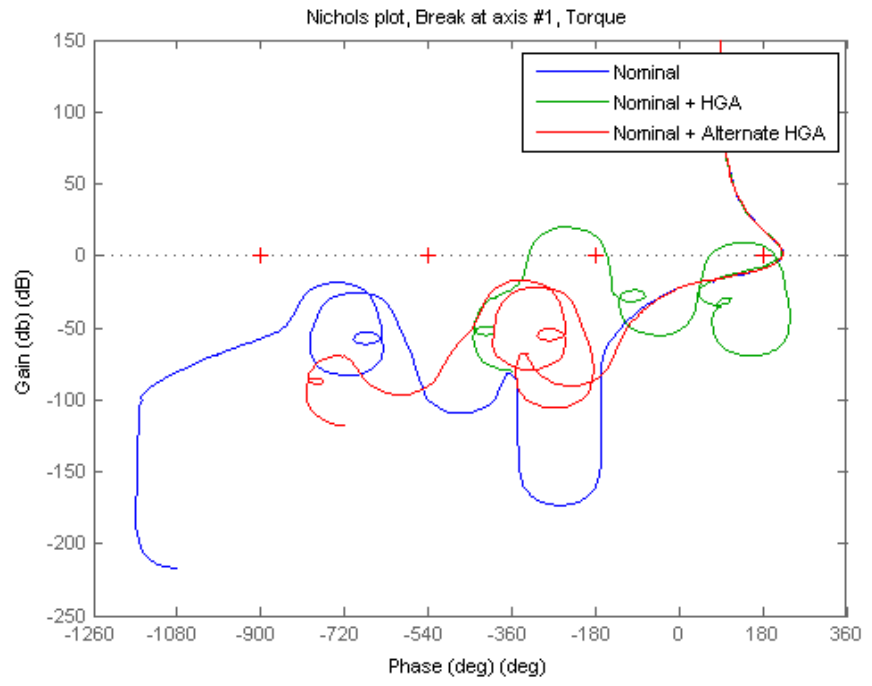

Figure 14. Nichols plot of HGA Control with $K_{\mathrm{FF}}=0$

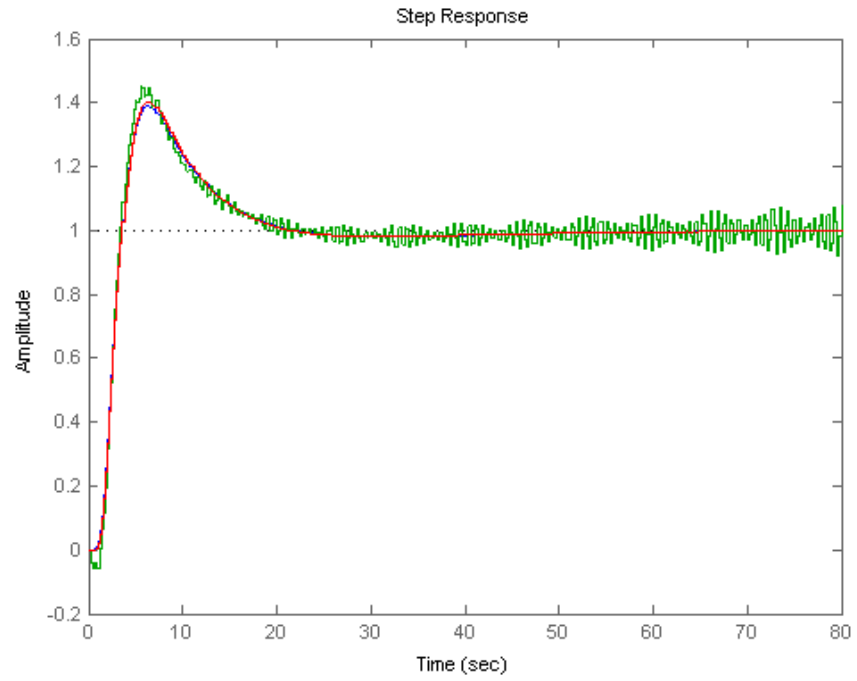

Figure 15. Step response of HGA Control with $K_{F F}=0$

The redesigned PI controller must have a higher bandwidth, to provide for improved tracking performance. Loopshaping design strategy is used, and the gains are selected based on:

$$
\begin{aligned}
& \zeta=0.8 \\
& \omega=0.02 \cdot 2 \cdot \pi \\
& K_{P}=2 \zeta \omega \\
& K_{I}=\omega^{2} \\
& K_{F F}=0
\end{aligned}
$$

Conducting a stability analysis on this new HGA controller yields the following characteristics, which is sufficient to meet all performance metrics. 
Bandwidth: $0.046 \mathrm{~Hz}$

Gain Margin: $27.9 \mathrm{~dB}$

Phase Margin: $65.9 \mathrm{deg}$

Settling Time: $40 \mathrm{sec}$

Figure 16, Figure 17, and Figure 18 show the effect of coupling the modified HGA controller with the spacecraft controller (blue line is nominal Observing mode without HGA, green is Observing mode with flight HGA, red is Observing mode with redesigned HGA control). The Bode plot shows that modal suppression is not completely restored (high frequency attenuation not as great with modified HGA control as with nominal Observing control), however much more than without the HGA flight controller. The Nichols plot shows that the high frequency flexible modes are attenuated and stability margins are maintained, and the closed loop step response shows stable behavior as compared to the nominal Observing mode controller and the Observing mode controller coupled with the flight HGA controller.

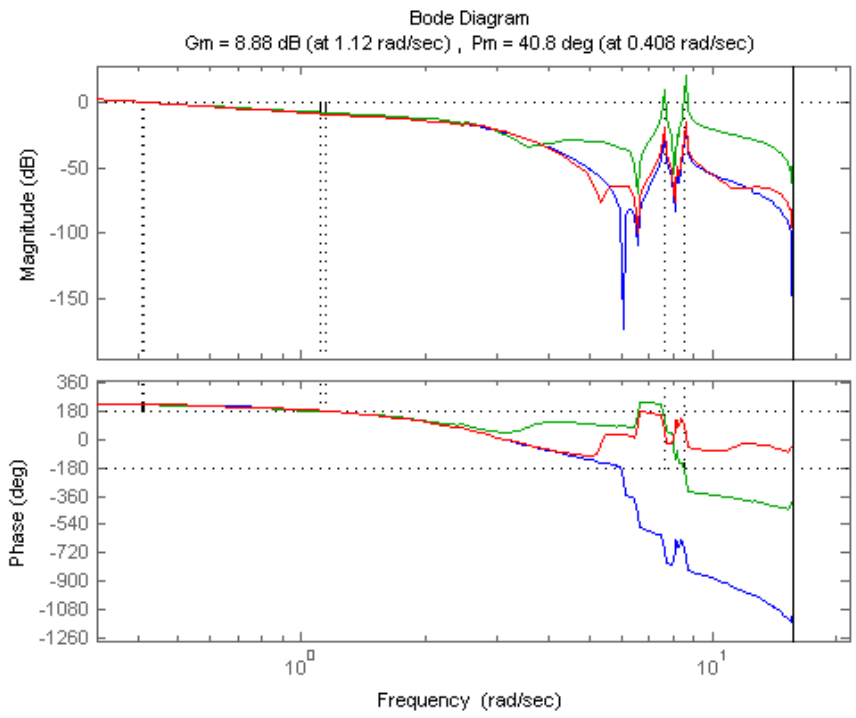

Figure 16. Bode plot of Modified HGA Control

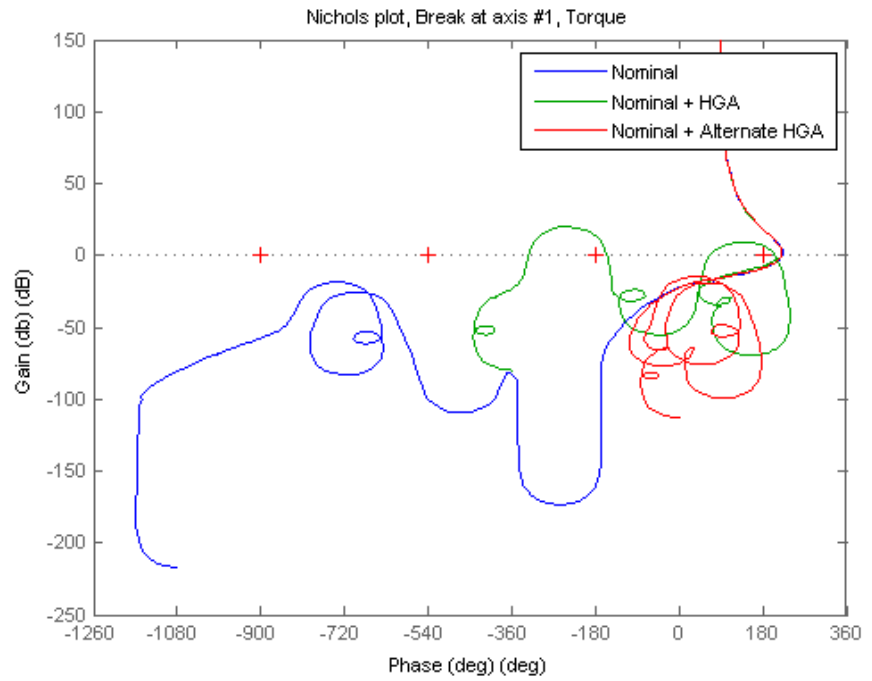

Figure 17. Nichols plot of Modified HGA Control 


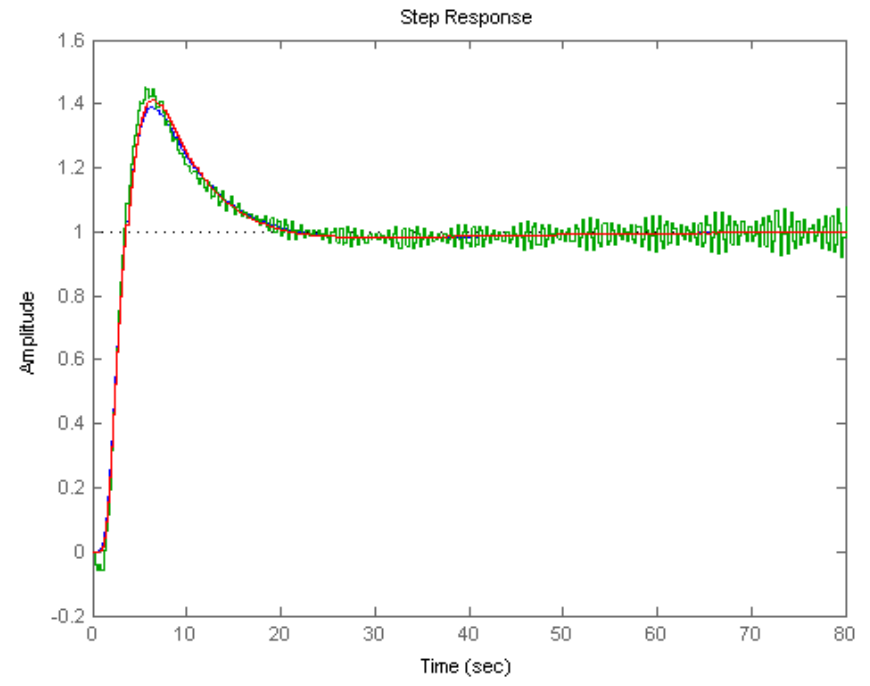

Figure 18. Closed Loop Step Response of Modified HGA Control

\section{CONCLUSION}

The LRO HGA Controller has performed well during the first 6-months of LRO's mission. However, during large spacecraft attitude maneuvers, communication is lost. This is caused by the fact that the HGA max rate is 0.5 $\mathrm{deg} / \mathrm{sec}$ while the spacecraft max rate is $0.1 \mathrm{deg} / \mathrm{sec}$, as well as the HGA angles being computed based on a target spacecraft attitude, instead of an estimated spacecraft attitude. This study sought to find a solution that would not require a change to flight software. A modified controller was designed that couples the HGA and spacecraft control loops through the estimated attitude. Coupling the controllers proved to cause stability issues. Therefore, the HGA controller gains were redesigned via loop-shaping techniques to yield a PI-controller that meets requirements. The modified controller showed to improved transient performance.

Future work involves conducting High Fidelity ( $\mathrm{HiFi}$ ) simulation runs to ensure the changes perform well. Prior to making these changes to the HGA control parameters, this analysis should be re-conducted with updated mass properties and flexible mode data based on best estimates and flight data. To advance the state of this work, a multivariable design and analysis approach could be taken.

This modification was proposed to the LRO Project as a solution to the loss of communication during spacecraft attitude maneuvers. Although shown to solve this problem, the LRO Project decided against implementing the proposed modification. 


\section{REFERENCES}

1. Lunar Reconnaissance Orbiter [Online]. - http://lroupdate.blogspot.com/

2. Garrick, J., "LRO Attitude Control System (ACS) Alignment and Coordinate Systems," 431-SPEC000653, April, 2009.

3. Garrick, J., "LRO Guidance Navigation and Control (GN\&C) Attitude Control System (ACS) Specifications," 431-SPEC-000162, February 2009.

4. Schepis, J.P., "Multipurpose Gimbal Actuator Performance Specification," 431-SPEC-000559, March 2007.

5. Broad Reach Engineering Company, "Starsys Gimbal Control Electronics Unit (GCE) For Lunar Reconnaissance Orbiter Project (LRO) User Guide,” DUDG10351000-14.

6. Shah, N. and Chen, J.R., "Inverse Kinematics Calibration Model for the Lunar Reconnaissance Orbiter (LRO)," Unpublished NASA TM.

7. Nise, N.S., Control Systems Engineering, Fourth Edition, John Wiley \& Sons, Inc., 2004.

8. Kuo, B.C. and Golnaraghi, F., Automatic Control Systems, Eighth Edition, John Wiley \& Sons, Inc., 2003.

9. Kane, T.R. and Levinson, D.A., Dynamics: Theory and Application, McGraw-Hill, Inc., 1985.

10. Vitale, J., and Roder, R., "Demiseable Integrated Reaction Wheel Assembly Wheel Speed Algorithm Specification," 431-SPEC-000843, November 2006.

11. Honeywell Inc., "Radiation Hardened Miniature Inertial Measurement Unit (MIMU) Intertial Reference Unit (IRU) Interface Control Document (ICD) for MIMU 1553B 28V Block 3 Program," ICDYG9666DG, May 2004. 